\title{
EDITORIAL
}

\section{Translational Issues in Neurorehabilitation}

Your guest editors for this issue received their graduate educations in neuroscience in the 1970s. We find it astonishing how much of what was then taught as fact or principle has since been proved wrong: Dale's law, for example (one neuron: one neurotransmitter), or the idea that gap junctions are rare and unimportant in the mammalian CNS. Diffusible neurotransmitters were unknown. Most spectacularly wrong was the dogma that plasticity and regeneration in the mammalian CNS vanish with maturation-that, once lesioned, the adult CNS structural and functional deficits are fixed and immutable.

This view began to change in the mid-1980s as Gage et al. ${ }^{1}$ found anatomical evidence that substantial plasticity occurs in adult brains after injury, and Merzenich et al. ${ }^{2}$ demonstrated functional reorganization of somatosensory maps in response to peripheral stimulation. The following decade yielded the insight that there is continuing production of neuroblasts in the paraventricular areas and that these cells can migrate throughout the brain, especially to areas of injury. ${ }^{3}$

This burgeoning of interest among the neuroscience community in the problems of recovery from neurological injury began to transform the clinical field of neurological rehabilitation from a sleepy backwater to one of vigorous investigation. A search on the NIH CRISP Web site of funded grants ${ }^{4}$ reveals that from 1977 to 1986 and from 1987 to 1996 only four new grants in each period were funded on the topic of "stroke rehabilitation." From 1997 to 2006, the number rose to 43. A PubMed" search on "neurological rehabilitation" yielded 244 articles published between 1977 to 1986, 904 published between 1987 to 1996, and some 1950 articles published between 1997 and the first half of 2006. Neurorehabilitation has come of age.

For this issue we wanted to highlight scientific areas that we consider central to the continuing development of the field, with articles that would serve both investigators and clinicians. Most of the work in this issue pertains to stroke, with only passing reference to other neurological conditions. It was not our intention to exclude other conditions, such as spinal cord injury, nor to concentrate so heavily on issues of stroke rehabilitation. That such a focus nonetheless emerged reflects the fact that investigations of recovery and rehabilitation of stroke in humans and animal models have preceded work on other conditions. Results from the first major random- ized controlled trial of a rehabilitation intervention for spinal cord injury were published only this year, in $2006 .^{6}$ Trials of interventions in traumatic brain injury have been difficult to accomplish, and agreement on appropriate animal models has been elusive. ${ }^{7}$ Research on rehabilitation for multiple sclerosis, Parkinson's disease, and other neurological conditions is even more sparse.

This issue begins, appropriately, with a review by Nudo on plasticity. In this review, he summarizes our understanding of the anatomical basis of plasticity in the cerebral cortex, local and remote changes in cortex in response to injury, and modulation of plasticity by behavioral training an adjuvant therapies. Two reviews follow (by Dromerick, Lum, and Hidler, and by Ivey, Hafer-Macko, and Macko), summarizing our current knowledge on the effects of different training regimens and rehabilitative strategies on recovery from stroke in humans. Dromerick et al. focus on strategies to promote recovery in hemiplegic stroke in the context of development and trial design for activity-based interventions; Ivey et al. focus on recovery of walking, aerobic fitness, and linkages with metabolism and cardiovascular risk factors.

The next set of articles explores the potential of adjuvant interventions, such as neurotransmitter agonists and antagonists, growth factors, and cell-based therapies, to potentiate the effects of training and exercise. Goldstein reviews the mechanisms and anatomical substrates responsible for the increase in cortical plasticity induced by amphetamines. Greenberg and Jin review the preclinical evidence suggesting that administration of growth factors can enhance cortical plasticity as well as clinical trials in humans to date. Chen and Chopp review prospects for enhancing plasticity beyond the acute period of injury.

How can we determine whether rehabilitation interventions are having a positive effect? We can, of course, measure the functional capacity of the patient, but it would be extremely valuable to have more rapidly responsive measures that provide feedback to clinicians and patients regarding the effects of specific exercises and interventions. Webster, Celnik, and Cohen review the data obtainable from transcortical magnetic stimulation, a noninvasive neurophysiological technique that may have therapeutic value in promoting plasticity, as well as providing maps of cortical excitability. Cramer 
reviews functional magnetic resonance imaging as a technique for imaging image recovery-in this context, imaging the areas of brain activated by specific tasks. Gan reviews the exciting prospects for use of two-photon imaging to measure cerebral plasticity in animal models.

Ultimately, therapies must be subjected to clinical trials, and those determined to be successful then need to be implemented in clinical practice. To obtain insight into indices of neurological recovery, Schallert reviews behavioral measurements employed in preclinical studies of recovery of function after neural injury. Barak and Duncan then provide a framework for understanding the problems of measurement in clinical trials of rehabilitation. They highlight the need for sensitive and appropriate outcome measures, review the problems with existing measures, and provide guidance on selection of appropriate measures for clinical trials. Chard reviews the evidence for community-based rehabilitation programs and provides a framework for developing research studies on implementation of programs and measurement of outcomes at the program and community levels.

We hope that these articles will serve as a useful collection of reference articles for both clinicians and investigators as they continue the development of the science and practice of neurorehabilitation. The field is still very young, and the needs are great, especially in light of the rapidly expanding population of elderly citizens in most countries. In reflecting upon where we stand at present, the words of Winston Churchill ${ }^{8}$ come to mind:
Now, this is not the end. It is not even the beginning of the end. But it is, perhaps, the end of the beginning.

\author{
Michael Chopp, Ph.D. \\ E-mail: chopp@neuro.hfh.edu \\ Michael Weinrich, M.D. \\ E-mail: weinricm@mail.nih.gov \\ Guest Editors
}

\section{REFERENCES}

1. Gage FH, Bjorklund A, Stenevi U. Cells of origin of the ventral cholinergic septohippocampal pathway undergoing compensatory collateral sprouting following fimbria-fornix transection. Neurosci Lett 1984;44:211-216.

2. Merzenich MM, Kaas JH, Wall J, et al. Topographic reorganization of somatosensory cortical areas $3 \mathrm{~b}$ and 1 in adult monkeys following restricted deafferentation. Neuroscience 1983;8:33-55.

3. Steindler DA, Kadrie T, Fillmore H, Thomas LB. The subependymal zone: "brain marrow." Prog Brain Res 1996;108:349-363.

4. U.S. National Institutes of Health. Computer Retrieval of Information on Scientific Projects (CRISP) [database online]. Available at: http://crisp.cit.nih.gov/. Updated weekly. Accessed Date: July 14, 2006.

5. U.S. National Library of Medicine. PubMed [database online] Available at: http://www.ncbi.nlm.nih.gov/entrez/query.fcgi? otool=nihlib. Updated daily. Accessed Date: July 14, 2006.

6. Dobkin B, Apple D, Barbeau H, et al. Weight-supported treadmill vs over-ground training for walking after acute incomplete SCI. Neurology 2006;66:484-493.

7. Narayan RK, Michel ME, Ansell B, et al. Clinical trials in head injury. J Neurotrauma 2002;19:503-557.

8. Churchill WS. Speech at the Lord Mayor's Luncheon, Mansion House, London. Nov. 10, 1942 [The New York Times. 1942 Nov. 11, p. 6]. 